\title{
SEM for the 21st Century: Scanning Ion Microscopy
}

\author{
David C. Joy
}

(C) ASM International. Reprinted with permission from Electronic Device Failure Analysis, 2012, 14(1), pp 4-12. 2012

\section{What Is Wrong with the Scanning Electron Microscope (SEM)?}

The scanning electron microscope (SEM) has become the most widely used of all advanced imaging tools because it offers a unique range of capabilities. It can resolve and image objects with sizes ranging from millimeters to below $1 \mathrm{~nm}$; it offers multiple ways to generate, collect, and display signals; the images produced contain information about the topography, chemical composition, and the magnetic, electrostatic, and crystallographic properties of the sample; and it can generate characteristic x-ray emission from the specimen to provide a quantitative chemical analysis. Unfortunately, one thing that it will be unable to do is maintain its competitive edge in the 21 st century.

This is because electrons are electromagnetic "waves" and thus the smallest spot, " $d$," into which an electron beam can be focused has a diameter of the order of:

$d=\lambda / \alpha$

where $\lambda$ is the wavelength of the electron, which for a typical SEM energy of $10 \mathrm{keV}$ is approximately $0.01 \mathrm{~nm}$, and $\alpha$ is the convergence angle of the focused beam (in radians). The resolution of the microscope is ultimately determined by the beam size, so high-performance imaging requires that " $d$ " be small; from Eq 1 this can only be achieved by making $\alpha$ larger. Unfortunately, all SEM lenses inherently suffer from electron-optical aberrations,

D. C. Joy $(\bowtie)$

University of Tennessee, Knoxville, Knoxville, TN, USA

e-mail: djoy@utk.edu

D. C. Joy

Center for Nanophase Materials Science, Oak Ridge

National Laboratory, Oak Ridge, TN, USA which result in the spot size always being bigger than the value predicted by Eq 1 by a factor that varies as $\alpha^{3}$. Any attempt to reduce the spot size by increasing the convergence angle will therefore actually increase its diameter and will simultaneously degrade the depth of field of the image (i.e., the distance along the beam for which the image is in focus), as this varies as $1 / \alpha$. It thus becomes increasingly difficult, and indeed ultimately impossible, to achieve the desired combination of high resolution and a large depth of field in an SEM [1].

\section{The Ion Beam Microscope}

The solution to this dilemma is to not use electrons at all but to instead employ a beam of helium $\left(\mathrm{He}^{+}\right)$or some other light ions. $\mathrm{A} \mathrm{He}^{+}$ion weighs $7800 \times$ more than an electron, so its wavelength, $\lambda$, is almost $100 \times$ smaller than that of an electron with the same energy. This fact now makes it possible to optimize optical performance. Because the wavelength is now much shorter, the beam convergence angle, $\alpha$, can also be reduced by a similar factor without incurring any increase in the spot size of the beam as the result of diffraction limiting (Eq 1). Because $\alpha$ is now so small, typically $<1 \mathrm{mrad}$ as compared to 15 or $20 \mathrm{mrad}$ in an SEM, the imaging depth of field, proportional to $1 / \alpha$, becomes much higher while the effect of the aberrations of the probe-forming lens, which scale as $\alpha^{3}$, become insignificant. The end result is thus a scanning microscope that is effectively free of aberrations and simultaneously provides a high spatial resolution and a large depth of field. These potential advantages of ion, compared to electron, beams for microscopy were recognized even before electron microscopes became commercially available, but it was not until the pioneering work of Professor Ricardo 
Levi-Setti at The University of Chicago in 1970 that highquality ion imaging — using protons $\left(\mathrm{H}^{+}\right)$-was first demonstrated [2]. Another 30 years of development was still required before ion sources with the brightness and stability necessary to compete head-to-head with field emission SEMs became available [3].

The general layout of an ion microscope (Fig. 1) is similar to that of an SEM, although the details are rather different. The beam is generated by a gaseous field ionization source (GFIS), a device that ionizes gaseous helium at an atomically sharp metal tip. The effective size of this source of ions is only about $0.3 \mathrm{~nm}$ in diameter, so no further demagnification is required to achieve a subnanometer-sized probe. The lenses, beam deflectors, and scanning system must be electrostatic rather than

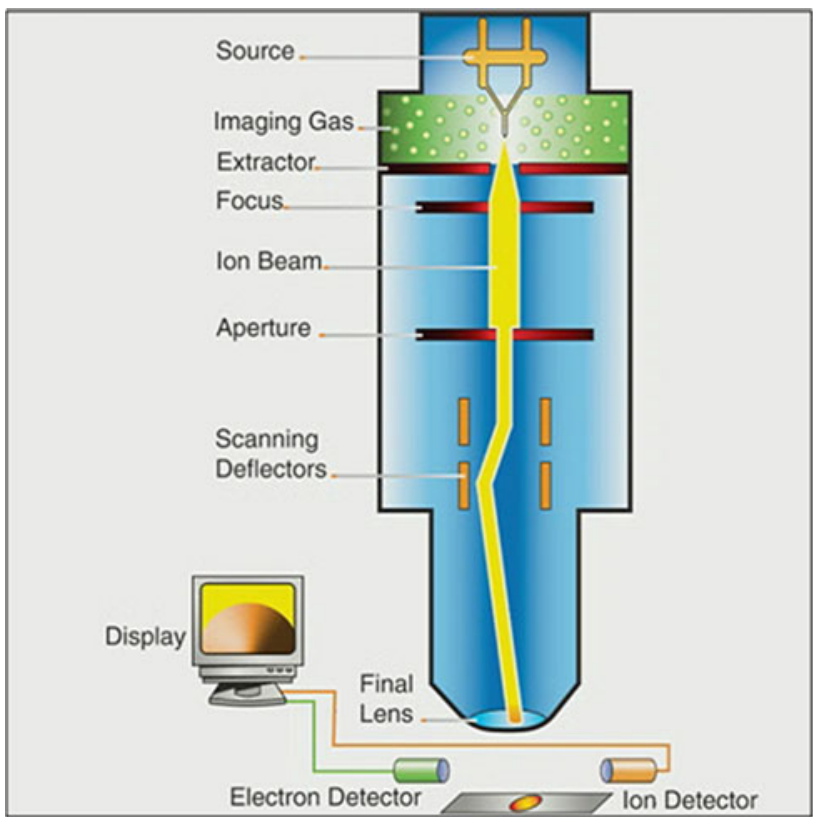

Fig. 1 Schematic diagram of a HIM showing the GFIS source, lenses, scan system, detector, and display electromagnetic in type because ions travel so slowly that they are only minimally affected by the Lorentz force from a magnetic field, a fact that also has the benefit of reducing the sensitivity of ion microscopes to stray fields in the laboratory. Finally, although the accelerating voltage of the helium ion microscope (HIM) may be numerically the same as that of an SEM, the properties of these two beams are very different. Because ions are much more massive than electrons, a $\mathrm{He}^{+}$ion with a kinetic energy of $30 \mathrm{keV}$ has only about the same velocity as a $3 \mathrm{eV}$ electron, so the concept of "low-voltage imaging" has quite a different meaning and implication for the two instruments.

\section{Secondary Electron Imaging with Ion Beams}

Both the HIM and the SEM can generate a secondary electron signal. A side-by-side comparison (Fig. 2) of secondary electron images of the same object but generated by ions (iSE) (Fig. 2a) and by electrons (eSE) (Fig. 2b) shows that the image information content and appearance are basically quite similar, although on closer examination, the iSE image is seen to contain more and higher-contrast surface detail. In fact, the iSE signal is readily capable of revealing even monolayer surface information with good visibility and contrast (Fig. 3). This is one result of an important difference between the way in which the iSE and eSE signals are generated. In the SEM, the secondary signal consists of two distinct components: SE1 secondary electrons, which are produced when the incident beam impinges on the surface of the sample, and SE2, which are generated by backscattered electrons (BSE) departing the sample. The SE1 signal contains high-resolution, surfacesensitive detail, but the SE2 signal, generated by BSEs emerging from a large area about the incident beam point, contains only low-resolution information about the subsurface interior of the sample. Because there are typically two SE2 electrons generated for every SE1, the visibility of surface detail in the composite eSE image is poor.
Fig. 2 Comparison of a $\mathrm{He}^{+}$ ion-generated iSE image and b electron-generated $\mathrm{eSE}$ image of the same region of a device structure. The iSE image was recorded at $30 \mathrm{keV}$ and the eSE image at $2 \mathrm{keV}$. Horizontal field of view $7 \mu \mathrm{m}$. Note the enhanced surface detail on the iSE compared to the eSE image
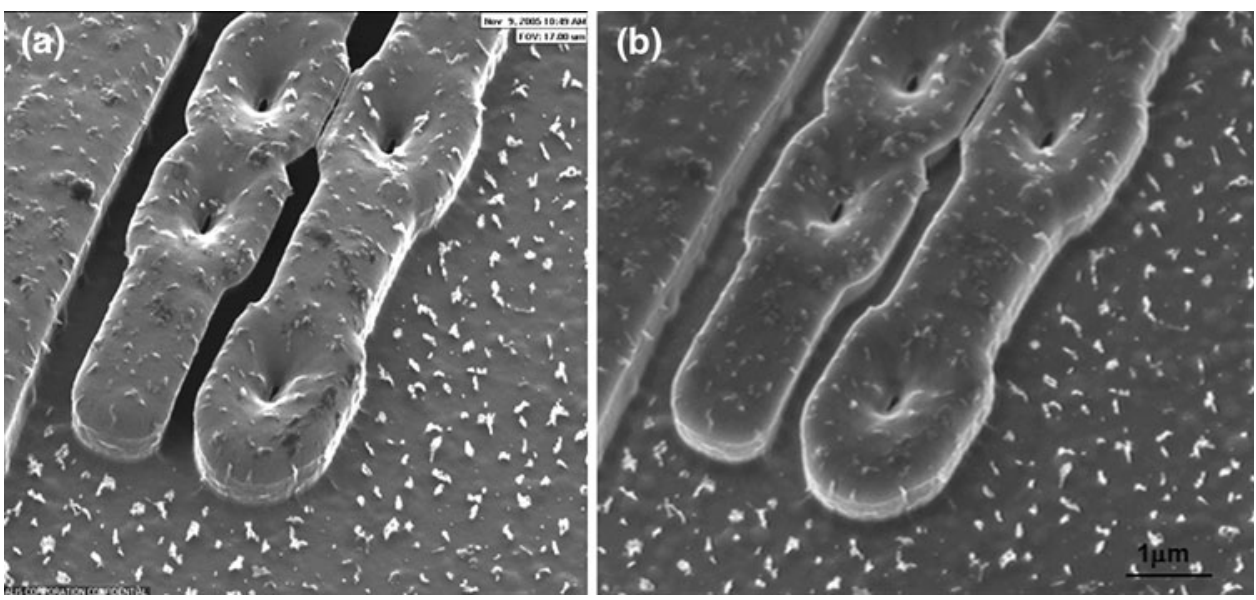


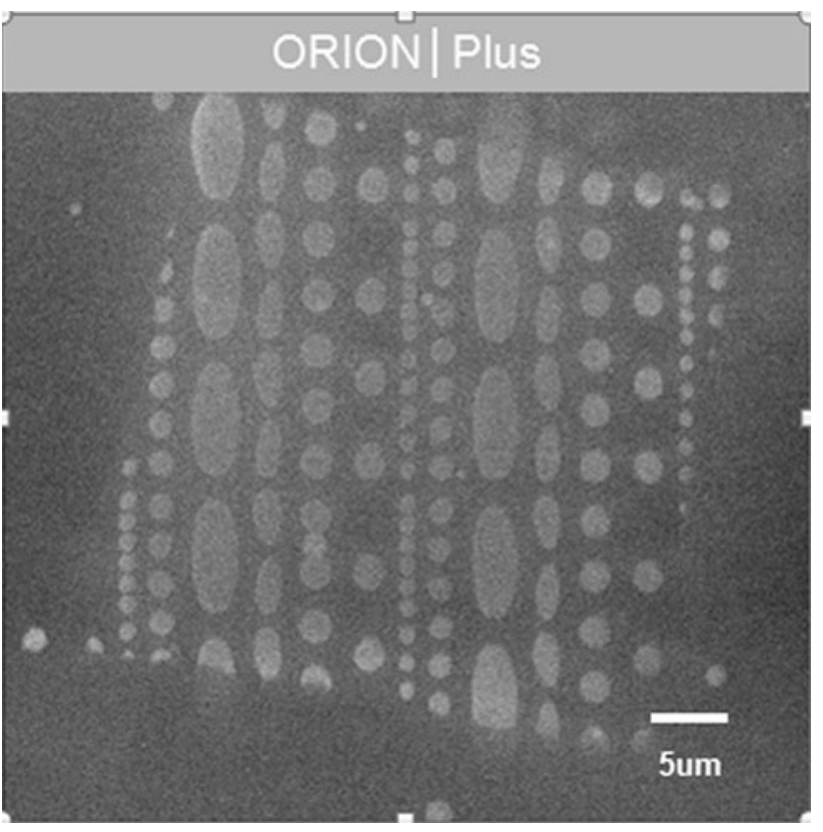

Fig. 3 iSE image of a self-assembled monolayer film of nitrobiphenyl-thiol (NBPT) on gold. Beam energy $38 \mathrm{keV}$

The HIM, on the other hand, produces only relatively few backscattered ions, and thus almost all the iSE signal is generated by the incident beam on the top surface of the sample. Consequently, surface contrast is more pronounced and resolution is enhanced. Other valuable imaging modes of the HIM, such as voltage contrast and dopant contrast, also benefit from the reduction or elimination of the backscatter-generated component.

In an SEM, the yield of secondary electrons per incident electron is low ( $<0.3$ eSE per incident electron) and falls as the beam energy is increased. In the HIM, the iSE yield is larger ( $>1$ iSE per incident electron) and rises further with increasing beam energy, so at typical SEM energies (20-30 keV), the ion beam current required to produce a high-quality SE image may be 10-20× smaller than the electron beam current required [4]. Consequently, it will always be advantageous to operate the HIM at its highest available energy because this maximizes the signal-tonoise ratio and further improves the resolution. Again unlike the situation in the SEM, raising the HIM beam energy does not greatly increase the range of the beam in the specimen. The range, $R_{\mathrm{e}}$ (in nanometers), of an electron beam of energy $E(\mathrm{keV})$ in a solid of density $\rho\left(\mathrm{g} / \mathrm{cm}^{3}\right)$ is given to a good approximation as [4]

$R_{\mathrm{e}}=78 \cdot E^{1.67} / \rho$

while for a $\mathrm{He}^{+}$ion, the corresponding range, $R_{\mathrm{He}}$ (nanometers), is

$R_{\mathrm{He}}=78 \cdot E^{0.7} / \rho$

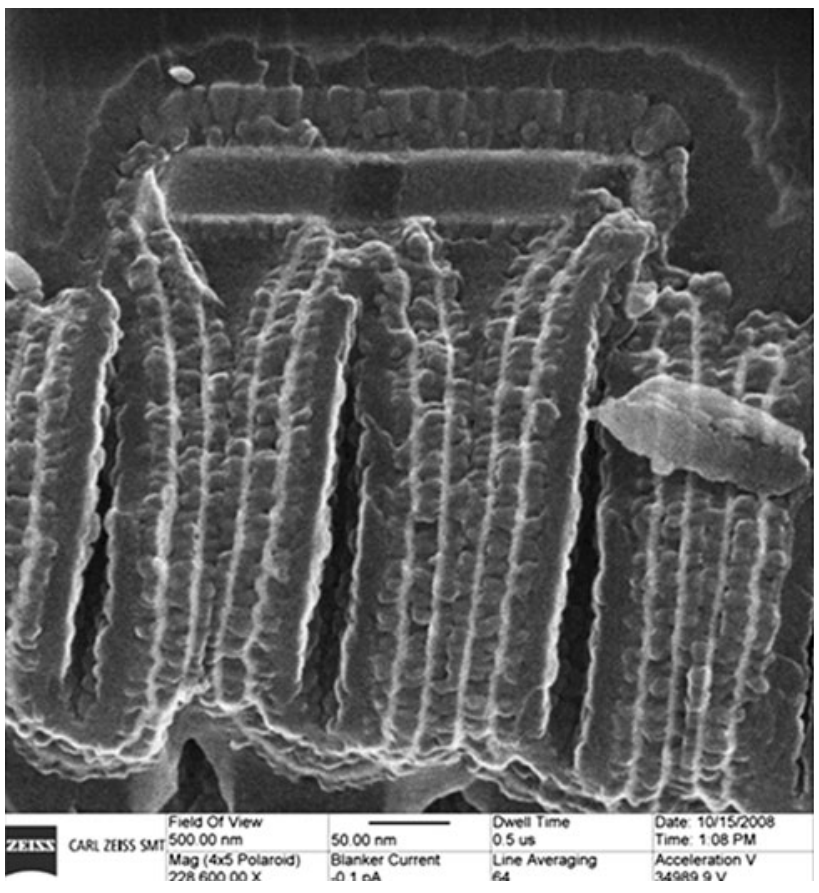

Fig. 4 iSE image of a cross sectioned semiconductor device structure. Horizontal field of view, $500 \mathrm{~nm}$. Note the high-resolution detail on the surfaces and the large depth of field. Beam energy $34.5 \mathrm{keV}$, beam current $0.1 \mathrm{pA}$, exposure time $40 \mathrm{~s}$

So, to a good approximation, the ratio of the ranges $R_{\mathrm{e}} / R_{\mathrm{He}}$ for any given energy $E$ is simply the numerical value of " $E$ "; i.e., at $10 \mathrm{keV}$ the electron beam range is $10 \times$ that of the $\mathrm{He}$ and $30 \times$ further at $30 \mathrm{keV}$. This result, and the fact that the iSE yield rises with an increase in accelerating voltage rather than falling as in the SEM, confirms that the future of the HIM will most likely be at energies substantially above the $40 \mathrm{keV}$ value that is now standard. Even without the benefit of higher beam energies, however, and as illustrated in Fig. 4, the combination of enhanced spatial resolution, depth of field, and signal-to-noise ratio that can be achieved at the $40 \mathrm{keV}$ level results in images that represent a major advance in scanning microscopy technique. In a future, fully optimized instrument operating at $150 \mathrm{keV}$ or even higher, it can be reasonably anticipated that single-atom imaging on the surfaces of bulk samples will be possible.

If heavier ions, such as $\mathrm{Ne}^{+}$or $\mathrm{Ar}^{+}$, are substituted in place of helium, there will be changes in many aspects of the performance of the scanning ion microscope. For example, ions that are heavier than helium can produce a larger yield of secondary electrons, but only at beam energies well above $100 \mathrm{keV}$. Under ideal conditions and at sufficiently high energies, heavy ions would be capable of achieving higher resolution because of their shorter wavelength, but, in practice, such ions often exhibit multiple ionization states $\left(1^{+}, 2^{+}\right.$, etc.). These effectively 
increase the energy spread of the ion beam, resulting in a larger, chromatically aberrated beam spot. Despite these issues, a scanning ion microscope in which it is possible to rapidly switch from one type of ion beam to another would permit light ions to be used for imaging and heavier ions to be employed for shaping specimens and removing contaminant layers, as well as sputtering material from the sample surface for microanalytical examination.

\section{Backscattered Ion Imaging}

Rutherford backscattered ions (RBI) are incident ions that have been backscattered from the specimen. These are comparable to the BSEs familiar in an SEM. Like BSE, the RBI yield, although usually quite small, tends to increase with the atomic number, $Z$, of the target and thus provides some chemical contrast in the image (Fig. 5). The RBI $Z$-contrast is usually more pronounced than that from BSEs; however, while it is useful in helping to visualize changes in sample composition, it is of little analytical value because the variation in the backscattered signal with atomic number is complicated and nonlinear [5]. The RBI signal from $\mathrm{He}^{+}$falls quite rapidly at energies higher than approximately $20 \mathrm{keV}$, thus limiting its value for imaging purposes. For heavier ions, the RBI yield rises with increasing beam energy until reaching a maximum value at typically a few hundred $\mathrm{keV}$ and then falling away.

\section{Is Microanalysis Possible in the HIM?}

One of the most popular and widely used applications of the modern SEM is its capability to generate a fluorescent $\mathrm{x}$-ray spectrum from the specimen under observation. Indeed, for many users, the ability to quickly answer the question "What in the world are we looking at?" is as or even more important than the production of a micrograph, so it is important to know whether an ion microscope could

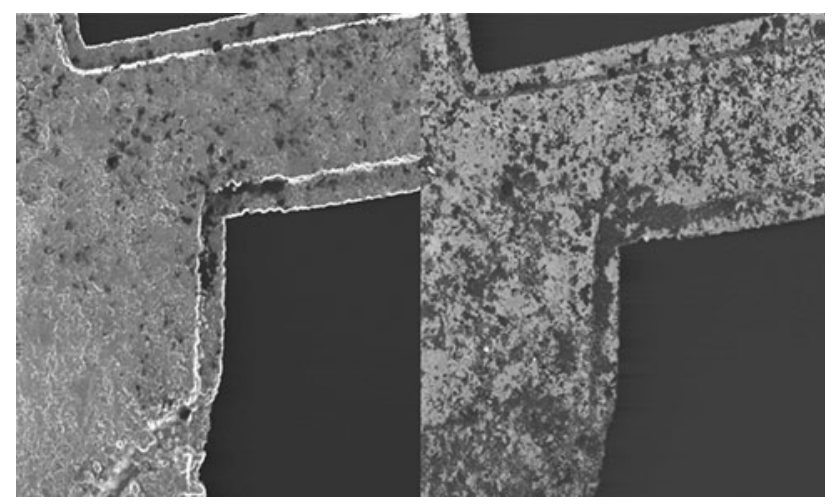

Fig. 5 Images of a copper grid with surface contaminated by small particles of carbon. a iSE signal and $\mathbf{b}$ RBI signal. Horizontal field of view $1 \mathrm{~mm}$, beam energy $38 \mathrm{keV}$ provide a similar capability. As first shown by Thompson in 1912 [6], a beam of electrons can generate x-rays from a material, provided that their velocity is equal to or greater than the orbital velocity of the inner shell electrons that are to be ionized. Because in an SEM the incident beam also consists of electrons, this is equivalent to saying that fluorescent $\mathrm{x}$-ray production can occur whenever the energy of the incident electrons $(\mathrm{keV})$ is equal to or greater than the ionization energy $(\mathrm{keV})$ of the electron shell of interest.

This statement is not correct when the incident beam consists of ions. Although these may have an energy that is equal to, or somewhat greater than, the ionization energy of the orbital shell of interest, their higher mass means that they are traveling much too slowly to be able to match their velocity with that of the orbiting electrons and thus instigate an ionization. For example, a $7 \mathrm{keV}$ electron beam can readily generate chromium $\mathrm{K}$-shell $\mathrm{x}$-rays that have an energy of approximately $5.5 \mathrm{keV}$. However, a helium beam would need energy of approximately $40 \mathrm{MeV}$ to match the electron orbital velocity and generate the K-shell emission. Therefore, $\mathrm{x}$-ray microanalysis is not a feasible mode of operation for an ion beam microscope, except under extreme conditions. Because microanalysis is of such importance for the majority of SEM users, some alternative microanalytical procedures must be devised.

As discussed earlier, the yield of RBI backscattered ions depends on the atomic number of the material, although not in a helpfully linear fashion. However, for a given angle of incidence of the ions onto the specimen and for a correspondingly well-defined take-off angle at which the backscattered ions are collected into a detector, there is a simple relationship between the incident energy of the ion, the energy with which it is scattered, and the scattering angle [7]. For example, if the scattering angle between the incident ion beam and the detector is $90^{\circ}$, then:

$E_{\text {scat }} / E_{0}=\left(M_{2}-M_{1}\right) /\left(M_{2}+M_{1}\right)$

where $E_{0}$ is the energy of the incident ion of mass $M_{1}, E_{\text {scat }}$ is the energy of the scattered ion, and $M_{2}$ is the mass of the element from which the scattering event occurred. In principle, this appears to be a simple and practical method for performing what is essentially energy-dispersive ion beam microanalysis. A solid-state diode detector similar to that used for energy-dispersive x-ray spectroscopy can be used to determine the energy of the scattered ion, $E_{\text {scat }}$. $M_{1}$ is known, and thus $M_{2}$ can be immediately determined. However, the variation of $E_{\text {scat }}$ as a function of $M_{2}$ is very nonlinear, so although peaks of low-atomic-number materials are easily separated from each other, at the other end of the periodic table the peaks are too closely packed to be distinguishable, given the current energy resolution of 
suitable solid-state detectors. In addition, unless the specimen is very thin (a few nanometers), a majority of the RBI will lose some amount of their energy within the sample as the result of inelastic scattering before they can escape and their energy can be measured. As a result, the energy $E_{\text {scat }}$ is no longer dependent solely on the atomic number of the target but varies depending on the thickness of the specimen. The elemental peaks therefore become difficult to uniquely identify because the broadening leads to overlaps and interferences with other peaks.

A promising alternative approach is that of secondary ion mass spectrometry, in which fragments of the specimen sputtered into the vacuum by the incident ion beam are collected and then characterized by measuring their massto-charge ratio. This approach was pioneered by Levi-Setti et al. [2, 8] in the 1960-1970. It is of value not only because it can perform analyses on just the top few atomic layers of a sample but also because of its overall high sensitivity and peak-to-background ratio, its excellent spatial resolution, and its potential range of applications, from isotopic analysis of elements to uniquely identifying polymers, cells, tissue, and other complex materials. Applying this technology to the HIM is an exciting possibility, although there are significant practical issues to overcome. The sputter yield (charged fragments emitted per ion) from helium beam is too small to provide adequate counting statistics from most materials within an acceptable time. However, if the ion microscope could rapidly switch from helium to heavier gases, such as neon or argon, then the $\mathrm{He}^{+}$can be reserved for high-resolution, lowdamage imaging while the heavy ions are employed for analytical purposes and to clean and modify surfaces.

\section{What Are the Problems with an HIM?}

In addition to all the benefits conferred by helium (or other ion beam) microscope, there are also two specific problem areas that must be considered: sample charging and the physical damage that can result from ion beam irradiation of a material.

\section{Charging}

Charging is a familiar problem in scanning electron microscopy when observing materials that are insulating or poor electrical conductors, a category that represents a high fraction of all materials of interest. To be able to image or perform an analysis on such materials, a variety of procedures have been developed, all of which, in one way or another, try to reach the condition at which the sample is charge-neutral and stable because it is emitting exactly as many electrons as it receives in a given time interval. In the early days of scanning microscopy, this was usually achieved by coating the sample with a metal film to enhance the emission of secondary electrons while placing a ground plane around the sample. Current practice often relies on adjusting the accelerating voltage to find the beam energy (usually denoted as E2) at which the total flux of secondary and BSEs emitted exactly equals the incident beam current, thus creating a dynamic charge balance. If the energy is set too high, the sample will acquire a net negative charge, and if the energy is too low, the specimen will charge positively.

Such methods do not work in the HIM, because when using a beam of positive ions, there is no charge balance condition. At all energies above a few $\mathrm{keV}$, each incident ion implants close to one unit of positive charge in the target and causes the emission of one or more secondary electrons, each carrying away one unit of negative charge. Poorly conducting specimens are therefore dosed with positive charge and depleted of electrons; they then charge positively, which reduces the emitted iSE signal and leads to an unstable landing energy for the beam, thus distorting the scan raster and varying the imaging magnification. The current solution to this problem is to periodically irradiate the scanned area with a broad beam of low-energy electrons at an intensity that is experimentally adjusted to dynamically maintain zero net potential in the field of view. This method works well even for highly insulating materials (Fig. 6), although, at least in theory, the addition

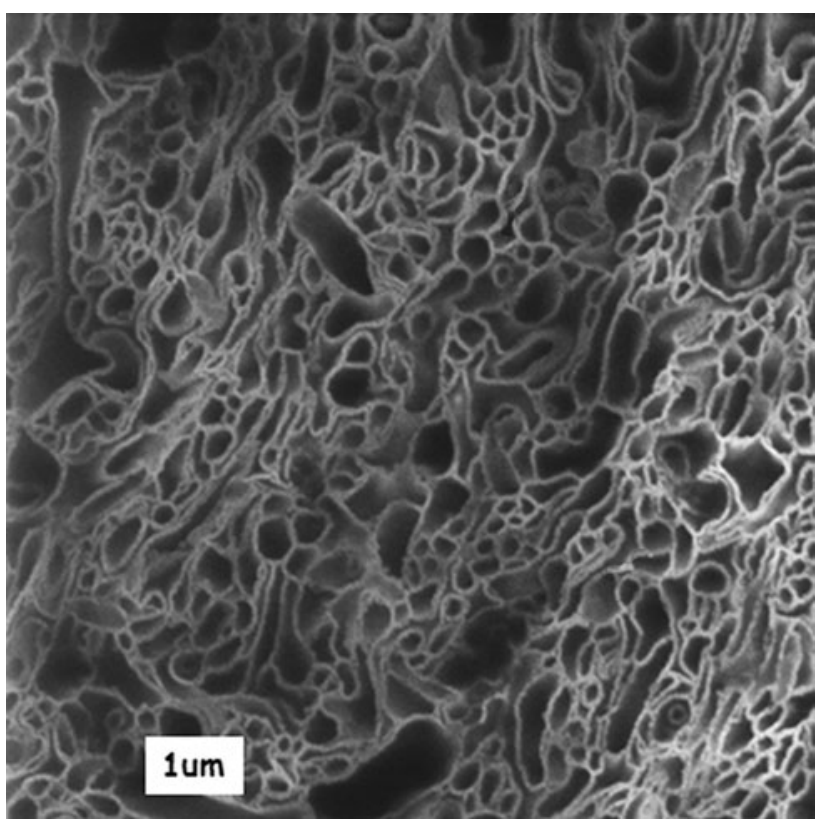

Fig. 6 iSE image of uncoated mammalian retinal tissue. Beam energy $38 \mathrm{keV}$. Charging has been stabilized by the use of a lowenergy electron flood gun 
of extra radiation may result in additional damage to radiation-sensitive samples.

\section{Ion Beam Damage}

The most commonly practiced application of ion beams is in focused ion beam instruments, where they are used to cut, shape, polish, and thin materials for later observation in transmission, scanning, or scanning transmission electron microscopes. This familiar application would necessarily seem to imply that the ion beams used for imaging and analytical purposes would also damage or destroy specimens while they are being imaged. Damage is most usually the result of sputtering, in which an incident ion transfers sufficient energy to an atom on the surface of the specimen to physically dislodge it. The yield of sputtered ions depends on the choice and energy of the incident ion beam as well as on the chemistry of the target material. It is lowest for light ions $\left(\mathrm{H}^{+}, \mathrm{He}^{+}\right)$and higher for heavier ions (Fig. 7). In general, the sputter yield rises with the energy of an ion until ultimately reaching a maximum and then starting to fall. In the case of the lightest ions $\left(\mathrm{H}^{+}, \mathrm{He}^{+}\right)$, however, the sputter yield rate starts small and falls still more as the energy is increased. This is significant because, as noted earlier, the iSE yield generated by a $\mathrm{He}^{+}$beam rises with beam energy. Consequently, the signal-to-noise ratio improves at higher energies, allowing the incident beam current to be reduced while maintaining a constant signal-to-noise ratio and also further reducing the sputter yield. So, at typical imaging currents of a few picoamps and beam energies of the order of $40 \mathrm{keV}$ and above, highquality imaging of most samples is readily possible without

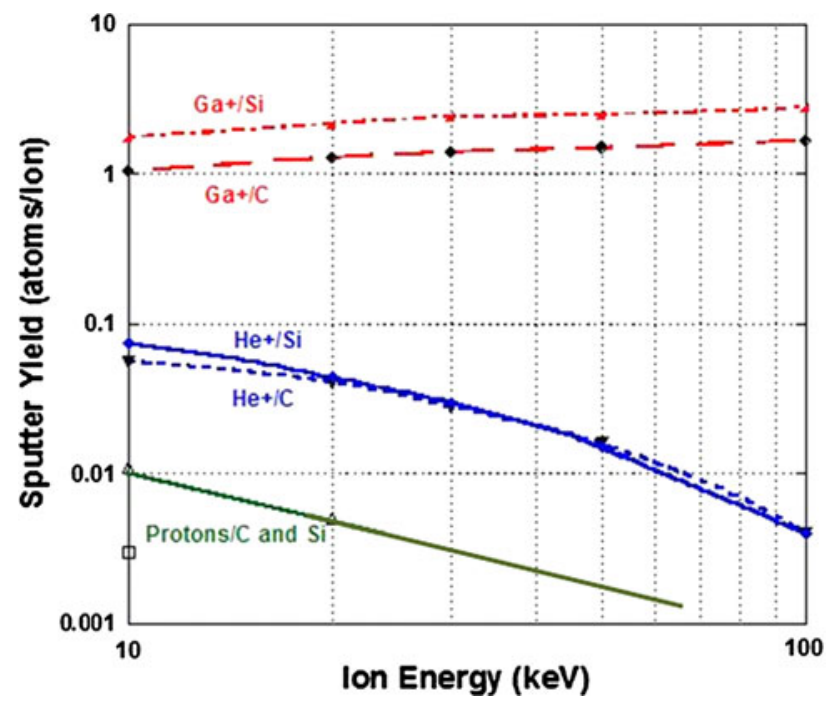

Fig. 7 Sputter generation rates for $\mathrm{H}^{+}, \mathrm{He}^{+}$, and $\mathrm{Ga}^{+}$beams on carbon and silicon surfaces and as a function of beam energy any fear of radiation damage occurring. However, radiolysis, which in rigidly bonded materials such as polymers causes mass loss and ultimately renders the material amorphous, is always as much of a problem as it is for electron beams and can only be managed by minimizing the beam dose.

With ions such as $\mathrm{Ar}^{+}$and $\mathrm{Ne}^{+}$, sputter damage is inevitable. However, if the instrument can be set up to rapidly switch between different beam sources, this is potentially an opportunity rather than a problem, because heavier ions can be used to clean surfaces and cut cross sections of samples, while all imaging can be performed with light ions.

\section{Summary}

SEM has made and will continue to make major contributions to imaging science and technology, but because of the comparatively large wavelength of $\mathrm{keV}$-energy electrons, SEMs cannot simultaneously offer high spatial resolution and a high depth of field. This is a major problem for nanoscience, biological, and all other fields of research where it must be assumed that all samples are threedimensional in nature. Ion beam microscopy can offer the promise of higher resolution imaging and overcome the problem of depth of field while also providing significantly more surface detail, a wide range of novel and familiar contrast mechanisms, and the potential for new microanalytical techniques that combine nanometer spatial resolution and single monolayer sensitivity.

Acknowledgments The author is grateful to Dan McGee and the HIM group at Carl Zeiss SMT for permission to use some of their images and for their unfailing help and enthusiasm, and to Professor Brendan Griffin (University of Western Australia) and Dr. Dale Newbury (National Institute of Standards and Technology) for valuable discussions. This work has been partially supported by SRC, Contract Manager Dr. D. Herr.

\section{References}

1. J. Goldstein, D.E. Newbury, D.C. Joy, C. Lyman, P.E. Echlin, E. Lifshin, L. Sawyer, J. Michael, Chap. 1-3, Scanning Electron Microscopy and X-Ray Microanalysis, 3rd edn. (Kluwer Academic/Plenum Publishers, New York, 2003)

2. R. Levi-Setti, Proton scanning microscopy: feasibility and promise, in Scanning Electron Microscopy, Part 1, ed. by O. Johari (IITRI, Chicago, 1974), pp. 125-135

3. R. Hill, J. Notte, B. Ward, The ALIS He ion source and its application to high resolution microscopy. Phys. Procedia 1, 135-141 (2008)

4. R. Ramachandra, B. Griffin, D.C. Joy, A model of secondary electron imaging in the helium ion scanning microscope. Ultramicroscopy 109, 748-757 (2009)

5. D.C. Joy, B.J. Griffin, Is microanalysis possible in the helium ion microscope? Microsc. Microanal. 17, 643-649 (2011) 
6. J.J. Thomson, Ionization by moving particles. Philos. Mag. 28, 250-262 (1912)

7. J.R. Tesmer, M. Nastasi, Handbook of Modern Ion Beam Materials Analysis (Materials Research Society, Boston, 1995)
8. R. Levi-Setti, G. Crow, Y.L. Wang, Progress in high resolution scanning ion microscopy and secondary ion mass spectrometry imaging microanalysis, in Scanning Electron Microscopy, Part 2, ed. by O. Johari (IITRI, Chicago, 1985), pp. 535-551 